\title{
Global Equity in Scholarly Communication
}

Johanna Havemann

Potential competing interests: The author(s) declared that no potential competing interests exist.

The opportunity for researchers from around the world being able to consume and share research output based on Open Science, FAIR and CARE principles. Since research capacity varies drastically within and across world regions, local conditions such as available funding, research infrastructure, or internet connectivity should not interfere with the potential of academic success. 